\title{
Comparison between trabectedin and doxorubicin in soft-tissue sarcomas: a systematic review and meta-analysis
}

\author{
Jingyi Dang"^ ${ }^{\#}$ Jun Fu", Zhao Zhang, Dong Liu, Debin Cheng, Hongbin Fan \\ Department of Orthopedic Surgery, Xijing Hospital, The Fourth Military Medical University, Xi'an, China \\ Contributions: (I) Conception and design: H Fan; (II) Administrative support: D Cheng; (III) Provision of study materials or patients: D Liu; (IV) \\ Collection and assembly of data: Z Zhang; (V) Data analysis and interpretation: J Fu, J Dang; (VI) Manuscript writing: All authors; (VII) Final \\ approval of manuscript: All authors. \\ "These authors contributed equally to this work. \\ Correspondence to: Hongbin Fan. Department of Orthopedic Surgery, Xijing Hospital, The Fourth Military Medical University, Xi'an, China. \\ Email: fanhb@fmmu.edu.cn.
}

\begin{abstract}
Background: This study sought to evaluate the differences between trabectedin and doxorubicin in the treatment of soft-tissue sarcoma (STS).

Methods: Multiple databases, including PubMed, Web of Science, Cochrane Library, and China National Knowledge Infrastructure, were searched to retrieve relevant articles. Ultimately, the full text of 10 studies involving the use of trabectedin and doxorubicin in STS were reviewed. Review Manager 5.2 was used to evaluate the heterogeneity of the results of the selected articles. Forest plot, bias, and sensitivity analyses were carried out on the included articles.

Results: Ten papers that met the criteria were included in this analysis. STS patients receiving trabectedin had longer progression-free survival than those receiving doxorubicin [overall mean difference $(\mathrm{MD})=1.36$, $95 \%$ confidence interval (CI): $1.04,1.68, \mathrm{I}^{2}=6 \%$, fixed-effects model]. The experimental group also had a longer overall survival period than the control group ( $\mathrm{MD}=3.92,95 \% \mathrm{CI}: 0.23,7.60, \mathrm{P}=0.04$ and $\mathrm{I}^{2}=83 \%$, random-effects model), and the experimental group had a better disease control rate than the control group (relative risk $=1.2, \mathrm{P}=0.03$ and $\mathrm{I}^{2}=45 \%$, fixed-effects model). From the publication bias analysis and sensitivity analysis, we can guarantee the results are robust and unbiased.

Discussion: Our research showed that STS patients who received trabectedin had better clinical effects and a longer survival time than those who received doxorubicin.
\end{abstract}

Keywords: Trabectedin; doxorubicin; soft tissue sarcoma (STS); meta-analysis

Submitted Oct 18, 2021. Accepted for publication Dec 06, 2021.

doi: $10.21037 /$ atm-21-6033

View this article at: https://dx.doi.org/10.21037/atm-21-6033

\section{Introduction}

Soft tissue sarcoma (STS) consists of rare malignancies, and accounts for about $1 \%$ of all adult cancers (1). There are more than 70 different histological STS subtypes; however, the most common high-grade STS subtypes include undifferentiated STS, liposarcoma, and smooth muscle sarcoma $(1,2) .60 \%$ of patients have local diseases at the time of diagnosis, and about $40 \%$ of patients develop metastasis within 5 years, which is associated with inferior survival outcomes (3). According to the medical history, clinical manifestation and laboratory examination, soft tissue sarcoma is not difficult to diagnose. The possibility

\footnotetext{
^ ORCID: 0000-0001-9127-6472.
} 
of this disease should be highly suspected in the following cases: the patient found painless progressive mass, fever and weight loss in a few weeks or months. Treatment options for STS include surgical resection, radiotherapy and drug therapy $(2,3)$.

For patients with localized STS, surgery is the main treatment option and has a potential curative effect (3). Other STS treatments include radiotherapy and chemotherapy (4). Anthracyclines (such as doxorubicin) are the common first-line drugs used in unresectable or metastatic STS. The median progression-free survival (PFS) time after doxorubicin treatment is 4 months (5). The median overall survival (OS) time is $12-18$ months after doxorubicin monotherapy (5-7).

Forty years after its emergence as a sarcoma treatment drug, Adriamycin remains the standard first-line treatment for STS. Randomized studies comparing doxorubicin to trabectedin did not find that the combined treatment group had a survival advantage, but a slight improvement in response rate was observed (8-10). Thus, the efficacy expectation of STS as a first-line system treatment is low.

In September 2007, trabectedin, the first anti-cancer derivative drug, was approved by the European Drug Administration to treat patients with advanced sarcoma after treatment with Doxorubicin has failed $(11,12)$. At present, trabectedin is used in nearly 80 countries worldwide to treat adult advanced STS after the failure of anthracycline and ifosfamide treatments, or to treat those who cannot be treated with anthracycline and ifosfamide $(13,14)$. In 2015, trabectedin was approved by the United States Food and Drug Administration after a critical randomized phase III trial with patients with advanced liposarcoma or leiomyosarcoma who did not respond to anthracyclinecontaining chemotherapy (14). trabectedin has a pleiotropic mechanism (15). In addition to its ability to induce the direct growth inhibition and death of malignant cells, trabectedin also has selective anti-inflammatory, immune regulating, and anti-angiogenetic characteristics. The safety of trabectedin is acceptable and manageable, and there is no evidence of cumulative toxicity or end-organ dysfunction even among patients who undergo long-term treatments with trabectedin (16-18). It was reported that the most common adverse reactions were nausea, fatigue, vomiting, constipation in drug treatment of STS (17).

To date, very few comprehensive analyses have been conducted comparing the effects of trabectedin and doxorubicin in the treatment of STS. Thus, we conducted a meta-analysis based on relevant randomized control trials
(RCTs) to explore this issue.

We present the following article in accordance with the PRISMA reporting checklist (available at https://dx.doi. org/10.21037/atm-21-6033).

\section{Methods}

\section{Literature search strategy}

The PubMed, Excerpta Medica dataBASE, Cochrane library, and China National Knowledge Infrastructure databases were systematically searched from their inception to September 2021, with the keywords: (I) softtissue sarcoma OR metastatic soft-tissue sarcomas; (II) chemotherapy OR doxorubicin; (III) trabectedin. The search strategy involved medical subject headings and text words combined with the Boolean operator, "AND". The literature search was comprehensive, and had no language restrictions or publication status limitations. To maximize the specificity and sensitivity of the retrieval, the authors also checked the reference list of the retrieved studies to identify other relevant studies that have not been identified by the retrieval strategy.

\section{Study selection}

Both RCTs and retrospective trials were included in the analysis. To be eligible for inclusion in the meta-analysis, articles had to meet the following inclusion criteria: (I) have full-text availability; (II) comprise a population of patients with mean ages ranging from 10 to 100 years; (III) compare interventions of trabectedin and doxorubicin; (IV) include the comparators; and $(\mathrm{V})$ have a RCT or retrospective trial design. Studies were excluded from the meta-analysis if they met any of the following exclusion criteria: (I) examined other topics; (II) compared other interventions; (III) had unavailable data; and/or (IV) were duplicate publications.

\section{Data extraction and quality assessment}

Two of the authors independently screened the papers included in the final analysis and extracted the relevant data directly from the documents. The following data were extracted: the name of the primary author, the country of the study, the number of patients in the study, the number of participants in each condition, and the age of the patients. If the standard deviation (SD) was missing, but the baseline SD was reported, the missing SD was replaced with 
baseline SD. If the average value could not be obtained but the median was reported, the median was used.

The risk of bias of the included studies was assessed using Cochrane's risk of bias tool. Two authors conducted risk of bias assessments for all the included RCTs. If there was a disagreement, a 3rd author reviewed the assessments, and the authors engaged in discussion until a consensus was reached.

\section{Statistical analysis}

Review manager (version 5.2, Cochrane Collaboration, 2011) was used to evaluate the results of the selected studies. To measure the consistency of the effect size [mean difference (MD) or odds ratio (OR)], a fixed- or random-effects model was chosen for the meta-analysis based on the $\mathrm{I}^{2}$ value to calculate the combined estimated value between the 2 interventions. Chi-square and $\mathrm{I}^{2}$ statistical tests were used to test heterogeneity of included comparators. If $\mathrm{I}^{2} \leq 50 \%$, there was no or little heterogeneity between the studies, and the fixed-effects model was used for the analysis. If $\mathrm{I}^{2}>50 \%$, there was a certain degree of heterogeneity between the studies, and the random-effects model was used for the analysis. A funnel chart was used to assess the publication bias. For the analysis of the included studies, the Begg and Egg tests were used to check for funnel chart symmetry and publication bias. In addition, sensitivity analyses were carried out.

\section{Results}

\section{Search process}

A total of 952 titles and abstracts were identified using the electronic screening search strategy. Of these, 725 full-text articles met the primary eligibility criteria for assessment. After careful reading, 82 studies were found to meet the preliminary criteria. These 82 articles were also reviewed by a second author, and the agreement between the authors was excellent. Ultimately, 10 articles met the final inclusion criteria and were included in this present meta-analysis. Further information on the search process and inclusion and exclusion criteria are presented in Figure 1.

\section{Characteristics of the included studies}

Table 1 provides a comprehensive description of each trial included in the meta-analysis. All the 10 articles were RCTs
(19-28). The 10 articles were published between 2015 and 2021. These 10 articles comprised a total of 1,800 patients (999 in the experimental group and 801 in the control group).

\section{Quality assessment results}

The studies were assessed using the Cochrane risk of bias tool. Only 1 study had a selection bias problem, and only 1 study had a reporting bias problem. In view of the bias summary, there were limited problems in attrition bias, reporting bias, and other biases (Figures 2,3). The funnel plots of the effect size versus the standard error for the studies were quite symmetrical, suggesting an absence of publication bias and a small-study effect. Egger's test confirmed the absence of publication bias $(\mathrm{P}=0.245)$.

\section{Heterogeneity test results}

\section{Heterogeneity analysis of PFS}

To analyze the difference in PFS between the experimental and control groups, a meta-analysis was conducted to calculate the MD using the fixed-effects model based on the heterogeneity analysis. The overall MD was $1.36[95 \%$ confidence interval $(\mathrm{CI}): 1.04,1.68]$. The $\mathrm{P}$ value of the overall effect was $<0.0001, \mathrm{I}^{2}=6 \%$, which demonstrated that the difference of PFS between the experimental and control groups was significant. Patients who received trabectedin had longer PFS than those who received doxorubicin (see Figure 4).

\section{Heterogeneity analysis of OS}

A meta-analysis of the OS times of the 2 groups was conducted. The results showed that there was significant difference in OS $(\mathrm{MD}=3.92,95 \% \mathrm{CI}: 0.23,7.60, \mathrm{P}=0.04$ and $\mathrm{I}^{2}=83 \%$, random-effects model). The experimental (trabectedin) group had a longer OS time than the control (doxorubicin) group (see Figure 5).

\section{Heterogeneity analysis of the DCR}

A meta-analysis of the disease control rate (DCR) was also conducted. The results showed that there was significant difference in the DCR ( $\mathrm{RR}=1.2, \mathrm{P}=0.03$ and $\mathrm{I}^{2}=45 \%$, fixedeffects model), and the experimental group had a better DCR than the control group (see Figure 6).

\section{Results of sensitivity analysis and publication bias}

In this study, a total of 7 articles reported on PFS. The 


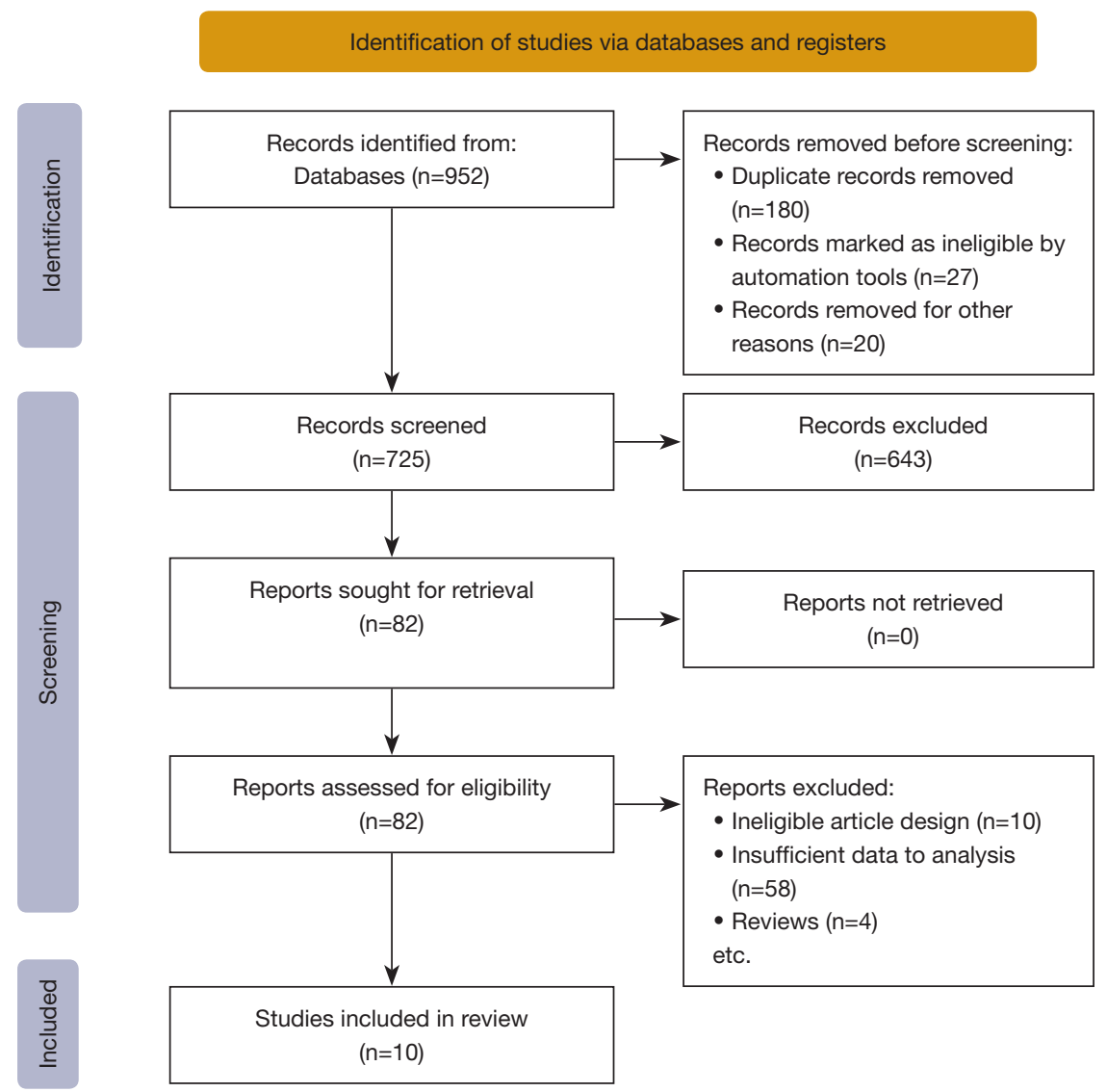

Figure 1 Flow diagram of literature search.

forest plot for PFS showed a significant difference between the 2 groups ( $\mathrm{MD}=1.36,95 \% \mathrm{CI}: 1.04,1.68, \mathrm{P}$ value of the overall effect: $<0.0001, \mathrm{I}^{2}=6 \%$; see Figure 4). We performed a sensitivity analysis by deleting the Schöffski (2021) study (26), and the $\mathrm{I}^{2}$ changed from $6 \%$ to $4 \%$ (see Figure 7 ), which indicated that the results of the included articles were robust. Finally, we used a funnel chart to evaluate the publication bias of the 2 groups in relation to PFS. As the Figure 8 shows, the graph is symmetrical. The $\mathrm{P}$ value of the Egger test was 0.24 , which indicates that there was no significant publication bias in this meta-analysis (see Figure 8).

\section{Discussion}

This analysis showed that trabectedin significantly increased the PFS of patients compared to doxorubicin, which is consistent with Chen's results. In this study, the OS time of the trabectedin group was considerably longer than that of the doxorubicin group $(\mathrm{P}<0.05)$, which indicates that trabectedin increases the OS time of STS patients. In addition, patients treated with trabectedin also had a better DCR than those treated with doxorubicin; thus, trabectedin had better clinical effects than doxorubicin in STS patients.

Mizuta's trial documented the efficacy of trabectedin in controlling advanced STS after previous cytotoxic chemotherapy failure (29). The patients studied underwent a great deal of pretreatment; however, the last systematic treatment, surgery, and radiotherapy failed, and their condition progressed rapidly. Compared to doxorubicin, the risk of disease progression or death in high-risk groups taking trabectedin was statistically reduced by $45 \%(\mathrm{P}<0.001)(30)$. The benefits of disease control can be observed regardless of a patient' disease histology or whether they had received previous systemic therapy. Notably, the most significant increase in the median PFS occurred in the liposarcoma subgroup. These findings are consistent with early findings on this unique and sensitive STS subtype, and recent reports on trabectedin activity in patients with translocation-related sarcoma (31). These findings provide further evidence that trabectedin plays a 
Table 1 Characteristics of included trials

\begin{tabular}{|c|c|c|c|c|c|c|}
\hline Study & Year & Type of study & Country & Intervention & $\mathrm{n}$ & Mean age (years) \\
\hline Cesne & & & & Best supportive care & 51 & 63.7 \\
\hline \multirow[t]{2}{*}{ Chawla } & 2015 & $\mathrm{RCT}$ & USA & Trabectedin & 83 & 54 \\
\hline & & & & Doxorubicin & 40 & 54 \\
\hline Demetri & & & & Dacarbazine & 173 & 56 \\
\hline \multirow[t]{2}{*}{ Hartmann } & 2020 & RCT & Germany & Trofosfamide & 80 & 70 \\
\hline & & & & Doxorubicin & 40 & 70.5 \\
\hline Hensley & 2015 & RCT & UK & Gemcitabine-docetaxel + trabectedin & 53 & 54.8 \\
\hline Jones & & & & Placebo + G/D & 70 & 54 \\
\hline \multirow[t]{2}{*}{ Martin-Broto } & 2016 & RCT & Spain & Trabectedin + doxorubicin & 54 & 53 \\
\hline & & & & Doxorubicin & 59 & 52 \\
\hline \multirow[t]{2}{*}{ Schöffski } & 2021 & $\mathrm{RCT}$ & Belgium & Trabectedin & 40 & 59.5 \\
\hline & & & & Dacarbazine & 40 & 56 \\
\hline \multirow[t]{2}{*}{ Seddon } & 2017 & $\mathrm{RCT}$ & UK & Trabectedin & 129 & 56 \\
\hline & & & & Dacarbazine & 128 & 55 \\
\hline Tian & 2020 & RCT & China & Trabectedin & 24 & $38.58 \pm 14.01$ \\
\hline
\end{tabular}

Random sequence generation (selection bias)

Allocation concealment (selection bias)

Blinding of participants and personnel (performance bias)

Blinding of outcome assessment (detection bias)

Incomplete outcome data (attrition bias)

Selective reporting (reporting bias)

Other bias

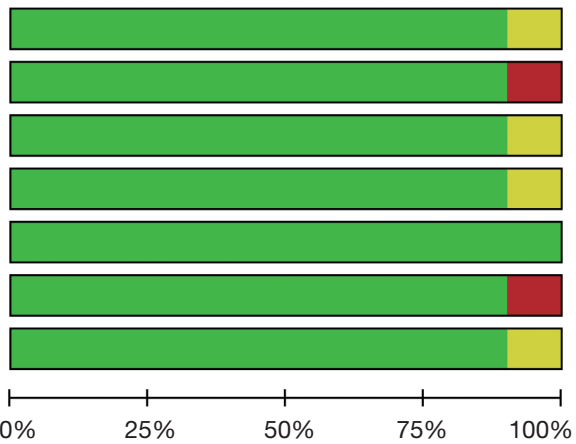

Low risk of bias

Unclear risk of bias

High risk of bias

Figure 2 Proportion of studies with low (green), high (red), or unclear (yellow) risk of bias. 


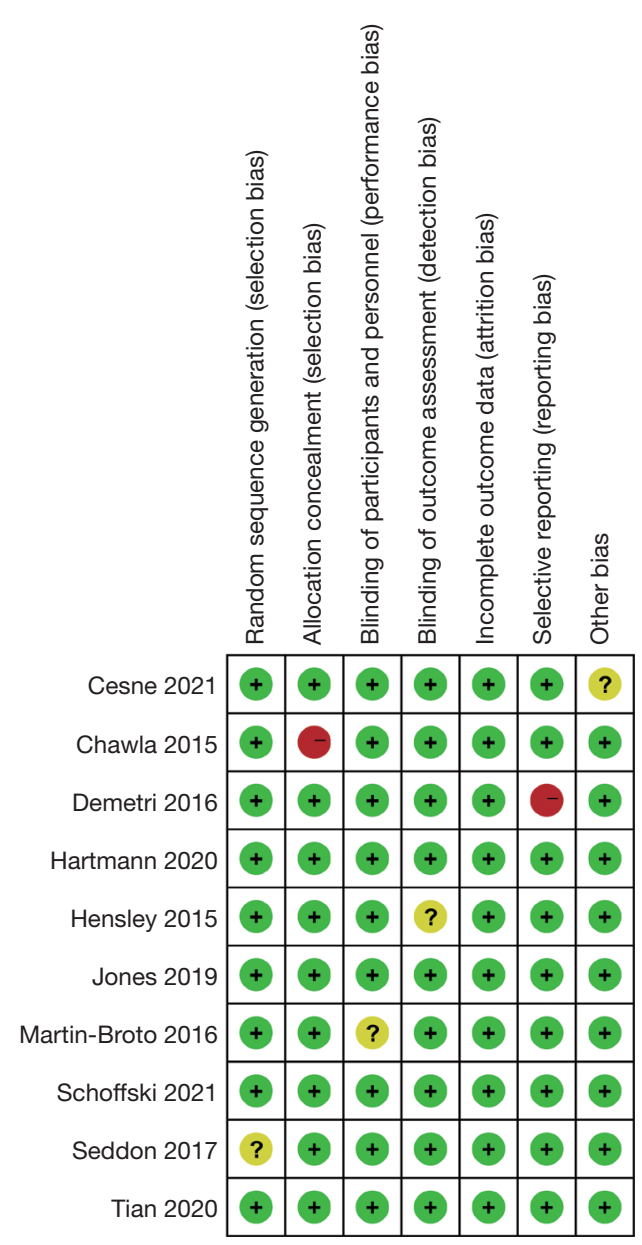

Figure 3 Risk of bias summary for included studies (red shading denotes a high risk of bias, yellow shading denotes an unclear risk of bias, and green shading denotes a low risk of bias). role as a direct inhibitor. Oncoproteins produced by fusion translocation produce transcriptional regulatory activity in these tumors. Our findings are also consistent with those of Mizut.

STS is a rare and diverse solid tumor that originates from mesenchymal precursors. STSs account for about $1 \%$ of all new adult malignancies (32). Doxorubicin, either alone or in combination with other chemotherapy, remains a standard treatment. However, the survival time of patients with metastatic diseases is only $12-16$ months, and the 2 -year survival rate is only about $30 \%(32,33)$.

In preclinical studies, the combination of trabectedin and doxorubicin has been shown to have a synergistic effect in sarcoma cell lines and tumor cells following human sarcoma xenotransplantation in mice (33). In these experiments, the order of first exposure to trabeculin and second exposure to doxorubicin proved to be more cytotoxic (34). Federica's trial could not exclude the potential advantages of trabectedin combined with doxorubicin in treating STS, such as leiomyosarcoma (35). In addition, there is convincing evidence of trabectedin activity in liposarcoma or leiomyosarcoma. Recent data confirm the effects of trabectedin even in more uncommon sarcomas $(36,37)$.

In conclusion, our research showed that patients who received trabectedin had better clinical effects and a longer survival time than those who received doxorubicin. However, this study had some limitations; for example, the included research areas should be expanded and more indicators for evaluating trabectedin in STS should be examined in the future.

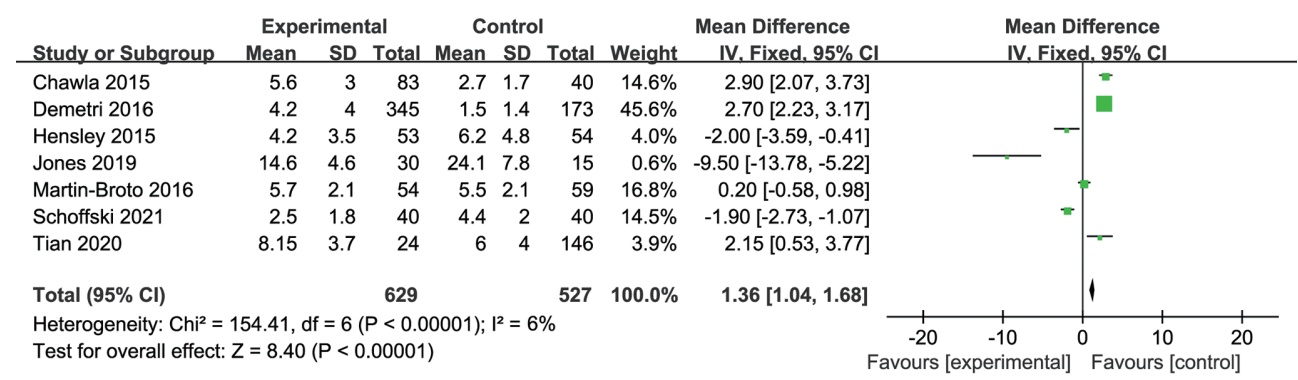

Figure 4 Forest plot of mean difference (MDs) with corresponding 95\% confidential intervals (CIs) in progression-free survival (PFS). 


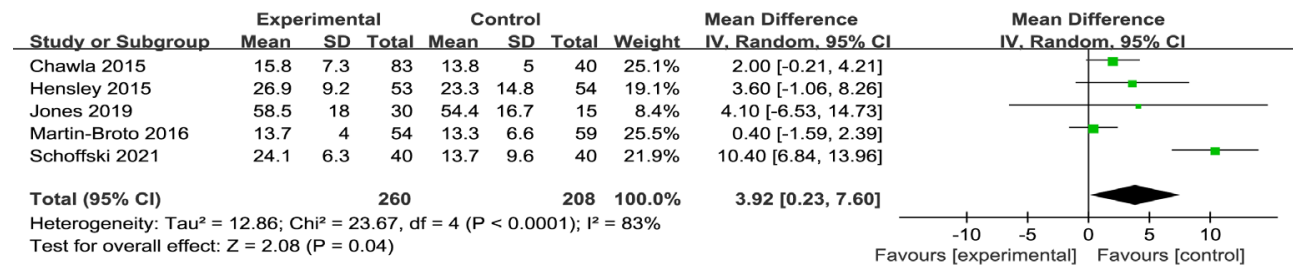

Figure 5 Forest plot of mean difference (MDs) with corresponding 95\% confidential intervals (CIs) in overall survival (OS).

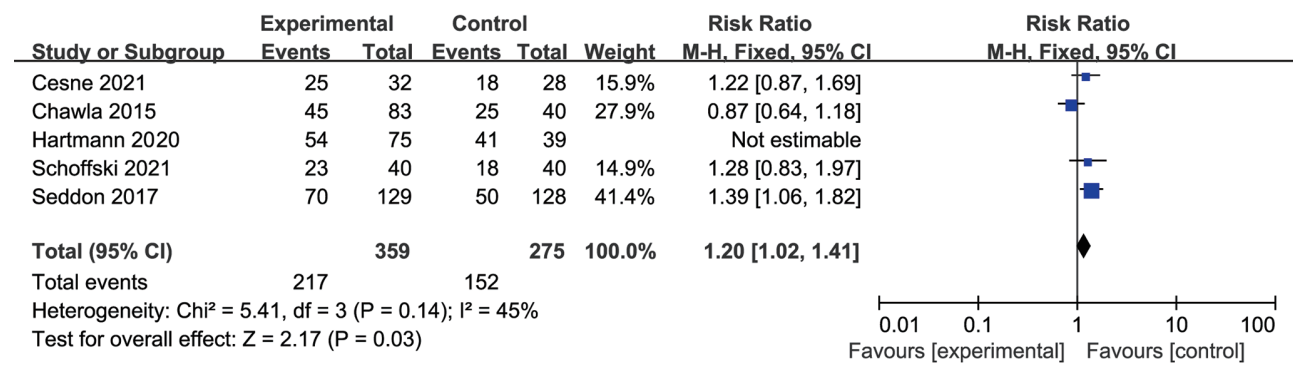

Figure 6 Forest plot of RRs with corresponding 95\% CIs in disease control rate (DCR).

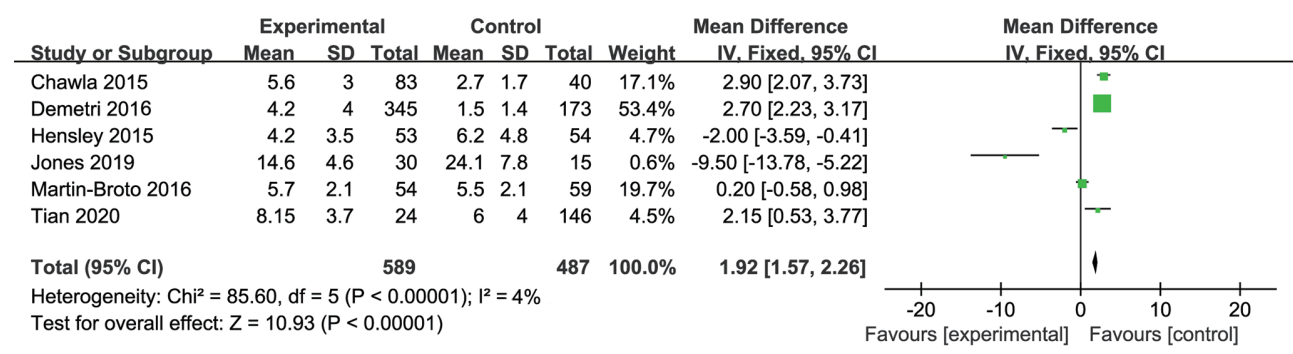

Figure 7 Sensitivity analysis of the forest plot of progression-free survival (PFS).

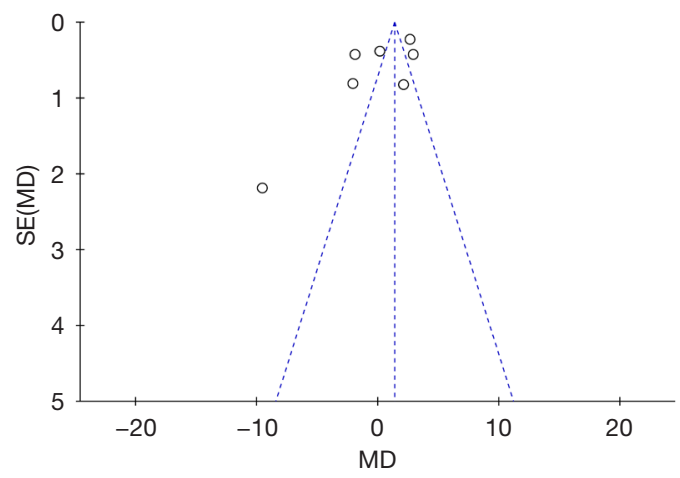

Figure 8 Funnel plot showing publication bias.

\section{Acknowledgments}

Funding: None.

\section{Footnote}

Reporting Checklist: The authors have completed the PRISMA reporting checklist. Available at https://dx.doi. org/10.21037/atm-21-6033

Conflicts of Interest: All authors have completed the ICMJE uniform disclosure form (available at https://dx.doi. 


\section{Page 8 of 9}

org/10.21037/atm-21-6033). The authors have no conflicts of interest to declare.

Ethical Statement: The authors are accountable for all aspects of the work in ensuring that questions related to the accuracy or integrity of any part of the work are appropriately investigated and resolved.

Open Access Statement: This is an Open Access article distributed in accordance with the Creative Commons Attribution-NonCommercial-NoDerivs 4.0 International License (CC BY-NC-ND 4.0), which permits the noncommercial replication and distribution of the article with the strict proviso that no changes or edits are made and the original work is properly cited (including links to both the formal publication through the relevant DOI and the license). See: https://creativecommons.org/licenses/by-nc-nd/4.0/.

\section{References}

1. Bokemeyer C, Franzke A, Hartmann JT, et al. A phase I/ II study of sequential, dose-escalated, high dose ifosfamide plus doxorubicin with peripheral blood stem cell support for the treatment of patients with advanced soft tissue sarcomas. Cancer 1997;80:1221-7.

2. Wang Y, Dai X, Zhu Y. Efficacy and safety of antiangiogenesis medicines for advanced soft tissue sarcoma: a meta-analysis. Transl Cancer Res 2020;9:1536-46.

3. Alvarez FJ, Kisseberth W, Hosoya K, et al. Postoperative adjuvant combination therapy with doxorubicin and noncytotoxic suramin in dogs with appendicular osteosarcoma. J Am Anim Hosp Assoc 2014;50:12-8.

4. Lori JC, Stein TJ, Thamm DH. Doxorubicin and cyclophosphamide for the treatment of canine lymphoma: a randomized, placebo-controlled study. Vet Comp Oncol 2010;8:188-95.

5. Davis EJ, Chugh R, Zhao L, et al. A randomised, openlabel, phase II study of neo/adjuvant doxorubicin and ifosfamide versus gemcitabine and docetaxel in patients with localised, high-risk, soft tissue sarcoma. Eur J Cancer 2015;51:1794-802.

6. Higuchi T, Miyake K, Sugisawa N, et al. Olaratumab combined with doxorubicin and ifosfamide overcomes individual doxorubicin and olaratumab resistance of an undifferentiated soft-tissue sarcoma in a PDOX mouse model. Cancer Lett 2019;451:122-7.

7. Wang L. Effect of Treadmill Intervention on Antitumor Effect and Cardiotoxicity of Doxorubicin. Genomics and
Dang et al. Meta-analysis of trabectedin and doxorubicin in STS

Applied Biology 2019;38:2318-22.

8. Hartmann JT, Horger M, Kluba T, et al. A noncomparative phase II study of dose intensive chemotherapy with doxorubicin and ifosfamide followed by high dose ICE consolidation with PBSCT in non-resectable, high grade, adult type soft tissue sarcomas. Invest New Drugs 2013;31:1592-601.

9. Villar VH, Vögler O, Martínez-Serra J, et al. Nilotinib counteracts $\mathrm{P}$-glycoprotein-mediated multidrug resistance and synergizes the antitumoral effect of doxorubicin in soft tissue sarcomas. PLoS One 2012;7:e37735.

10. Karch A, Koch A, Grünwald V. A phase II trial comparing pazopanib with doxorubicin as first-line treatment in elderly patients with metastatic or advanced soft tissue sarcoma (EPAZ): study protocol for a randomized controlled trial. Trials 2016;17:312.

11. Tanaka K, Mizusawa J, Fukuda H, et al. Adjuvant and neoadjuvant chemotherapy (NAC) with ifosfamide (IFO) and Doxorubicin hydrochloride (ADM) for high-grade soft tissue sarcomas (STS) in the extremities: Japan Clinical Oncology Group study JCOG030404. J Clin Oncol 2011;29:10078.

12. Yonemori K, Kodaira M, Satoh T, et al. Phase 1 study of olaratumab plus doxorubicin in Japanese patients with advanced soft-tissue sarcoma. Cancer Sci 2018;109:3962-70.

13. Kawaguchi K, Nakano K, Urasaki T, et al. Retrospective Analysis of Trabectedin Therapy for Soft Tissue Sarcoma. In Vivo 2019;33:1609-14.

14. Leporini C, Patanè $M$, Saullo F, et al. A comprehensive safety evaluation of trabectedin and drug-drug interactions of trabectedin-based combinations. BioDrugs 2014;28:499-511.

15. Casali PG, Sanfilippo R, D'Incalci $M$. Trabectedin therapy for sarcomas. Curr Opin Oncol 2010;22:342-6.

16. Blay JY. Trabectedin: An Emerging Therapeutic Option in Soft Tissue Sarcoma. European J Clin Med Oncol 2010;2:1-7.

17. Banerjee SN, Mitchell S, Al-Muderis O, et al. The Royal Marsden Hospital experience of Trabectedin in patients with advanced soft tissue sarcoma (STS): Toxicity and efficacy in a nonselected group. J Clin Oncol 2011;29:e20507.

18. Gronchi A, Hindi N, Cruz J, et al. Trabectedin and RAdiotherapy in Soft Tissue Sarcoma (TRASTS): Results of a Phase I Study in Myxoid Liposarcoma from Spanish (GEIS), Italian (ISG), French (FSG) Sarcoma Groups. EClinicalMedicine 2019;9:35-43.

19. Le Cesne A, Blay JY, Cupissol D, et al. A randomized 
phase III trial comparing trabectedin to best supportive care in patients with pre-treated soft tissue sarcoma: T-SAR, a French Sarcoma Group trial. Ann Oncol 2021;32:1034-44.

20. Chawla SP, Papai Z, Mukhametshina G, et al. First-Line Aldoxorubicin vs Doxorubicin in Metastatic or Locally Advanced Unresectable Soft-Tissue Sarcoma: A Phase 2b Randomized Clinical Trial. JAMA Oncol 2015;1:1272-80.

21. Demetri GD, von Mehren M, Jones RL, et al. Efficacy and Safety of Trabectedin or Dacarbazine for Metastatic Liposarcoma or Leiomyosarcoma After Failure of Conventional Chemotherapy: Results of a Phase III Randomized Multicenter Clinical Trial. J Clin Oncol 2016;34:786-793

22. Hartmann JT, Kopp HG, Gruenwald V, et al. Randomised phase II trial of trofosfamide vs. doxorubicin in elderly patients with untreated metastatic soft-tissue sarcoma. Eur J Cancer 2020;124:152-60.

23. Hensley RL, Monk BJ, Knoblauch RE, et al. A phase III study of trabectedin ( $\mathrm{T}$ ) plus pegylated liposomal doxorubicin (PLD) versus PLD for treatment of advancedrelapsed epithelial ovarian, primary peritoneal, or fallopian tube cancer. J Cli Onco 2015;33:1180-1186.

24. Monk BJ, Herzog TJ, Triantos S, et al. A randomized, open-label study comparing trabectedin and pegylated liposomal doxorubicin with pegylated liposomal doxorubicin alone for the treatment of advanced-relapsed epithelial ovarian, primary peritoneal, or fallopian tube cancer (ET743-OVC-3006). Gynecol Oncol 2019;154(Supplement 1):12-13.

25. Martin-Broto J, Pousa AL, de Las Peñas R, et al. Randomized Phase II Study of Trabectedin and Doxorubicin Compared With Doxorubicin Alone as FirstLine Treatment in Patients With Advanced Soft Tissue Sarcomas: A Spanish Group for Research on Sarcoma Study. J Clin Oncol 2016;34:2294-302.

26. Schöffski P, Toulmonde M, Estival A, et al. Randomised phase 2 study comparing the efficacy and safety of the oral tyrosine kinase inhibitor nintedanib with single agent ifosfamide in patients with advanced, inoperable, metastatic soft tissue sarcoma after failure of first-line chemotherapy: EORTC-1506-STBSG "ANITA". Eur J Cancer 2021;152:26-40.

27. Seddon B, Strauss SJ, Whelan J, et al. Gemcitabine and docetaxel versus doxorubicin as first-line treatment in previously untreated advanced unresectable or metastatic soft-tissue sarcomas (GeDDiS): a randomised controlled phase 3 trial. Lancet Oncol 2017;18:1397-410.

28. Tian Z, Yang Y, Yang Y, et al. High cumulative doxorubicin dose for advanced soft tissue sarcoma. BMC Cancer 2020;20:1139.

29. Mizuta Y, Tokuda K, Guo J, et al. Sodium thiosulfate prevents doxorubicin-induced DNA damage and apoptosis in cardiomyocytes in mice. Life Sci 2020;257:118074.

30. Endo M, Takahashi S, Araki N, et al. Time lapse analysis of tumor response in patients with soft tissue sarcoma treated with trabectedin: A pooled analysis of two phase II clinical trials. Cancer Med 2020;9:3656-67.

31. Schack LH, Mouritsen LS, Elowsson C, et al. The Danish experience with trabectedin treatment for metastatic sarcoma: Importance of hyponatremia. Acta Oncol 2015;54:34-40.

32. Almeida GF, Castro G Jr, Snitcovsky IM, et al. Sequential dose-dense Doxorubicin and Ifosfamide in advanced softtissue sarcoma patients in an out-patient-basis schedule. Sarcoma 2011;2011:984340.

33. Altieri P, Spallarossa P, Barisione C, et al. Inhibition of doxorubicin-induced senescence by PPAR $\delta$ activation agonists in cardiac muscle cells: cooperation between PPAR $\delta$ and Bcl6. PLoS One 2012;7:e46126.

34. Kawai A, Yonemori K, Takahashi S, et al. Systemic Therapy for Soft Tissue Sarcoma: Proposals for the Optimal Use of Pazopanib, Trabectedin, and Eribulin. Adv Ther 2017;34:1556-71.

35. Recine F, Bongiovanni A, Riva N, et al. Update on the role of trabectedin in the treatment of intractable soft tissue sarcomas. Onco Targets Ther 2017;10:1155-64.

36. De Sanctis R, Marrari A, Marchetti S, et al. Efficacy of trabectedin in advanced soft tissue sarcoma: beyond lipo- and leiomyosarcoma. Drug Des Devel Ther 2015;9:5785-91.

37. Duffaud F, Maki RG, Jones RL. Treatment of advanced soft tissue sarcoma: efficacy and safety of trabectedin, a multitarget agent, and update on other systemic therapeutic options. Expert Rev Clin Pharmacol 2016;9:501-12.

Cite this article as: Dang J, Fu J, Zhang Z, Liu D, Cheng D, Fan H. Comparison between trabectedin and doxorubicin in soft-tissue sarcomas: a systematic review and meta-analysis. Ann Transl Med 2021;9(24):1764. doi: 10.21037/atm-21-6033 